
ABHANDLUNGEN
DER AKADEMIE DER WISSENSCHAFTEN DER DDR

Abteilung Mathematik - Naturwissenschaften - Technik

Jahrgang $1988 \cdot$ Nr. $1 \mathrm{~N}$

\title{
Electrocardiology '87
}

Proceedings of the 14th International

Congress on Electrocardiology

Berlin, August 17th-20th, 1987

The Congress was organized by

The Society of Physiologists of the GDR under the auspices of the

International Council on Electrocardiology

Editor

Ernst Schubert

Assistant Editor

Dietrich Romberg 
Herausgegeben im Auftrag des Präsidenten

der Akademie der Wissenschaften der DDR

von Vizepräsident Prof. Dr. Heinz Stiller

The papers in this issue were printed without editorial reconsideration and therefore the responsibility of the contributions is exclusively with the authors.

ISBN 3-05-500541-4

ISSN 0138-1059

Erschienen im Akademie-Verlag Berlin, DDR-1086 Berlin, Leipziger Straße 3-4

(C) Akademie-Verlag Berlin 1988

Lizenznummer $202 \cdot 100 / 375 / 88$

Printed in the German Democratic Republic

Gesamtherstellung: VEB Druckerei „Thomas Müntzer“, 5820 Bad Langensalza

LSV 2075

Besstellnummer: $7638618(2001 / 88 / 1 \mathrm{~N})$

08800 
International Council on Electrocardiology
founded by P. W. Rijlant, Belgium

President

President Elect

Honorary Members
E. Schubert, GDR

B. Taccardi, Italy

H. Abel, FRG

P. d'Alche, France-

R. Amirov, USSR

Z. Antaloczy, Hungary

A. J. Bayes de Luna, Spain

K. Harumi, Japan

J. Jagielski, Poland

F. Kornreich, Belgium

P. Macfarlane, United KIngdom

H. PIpberger, USA

F. de Padua, Portugal

Z. Pavlov, Bulgaria

J. C. RIos, USA

P. Rautaharju, Canada

I. Ruttkay-Nedecky, CSSR

R. Selvester, USA

L.I. Titomir, USSR

H. Ueda, Japan

R. Th. van Dam, The Netherlands

R. Wenger, Austria 